\title{
The calcineruin inhibitor cyclosporine a synergistically enhances the susceptibility of Candida albicans biofilms to fluconazole by multiple mechanisms
}

Wei Jia ${ }^{1,2}$, Haiyun Zhang ${ }^{3}$, Caiyun $\mathrm{Li}^{4}$, Gang Li ${ }^{1,2}$, Xiaoming Liu ${ }^{1 *}$ and Jun Wei $\mathrm{i}^{1,2^{*}}$

\begin{abstract}
Background: Biofilms produced by Candida albicans (C. albicans) are intrinsically resistant to fungicidal agents, which are a main cause of the pathogenesis of catheter infections. Several lines of evidence have demonstrated that calcineurin inhibitor FK506 or cyclosporine A (CSA) can remarkably enhance the antifungal activity of fluconazole (FLC) against biofilm-producing C. albicans strain infections. The aim of present study is thus to interrogate the mechanism underpinning the synergistic effect of FLC and calcineurin inhibitors.

Results: Twenty four clinical C. albicans strains isolated from bloodstream showed a distinct capacity of biofilm formation. A combination of calcineurin inhibitor CsA and FLC exhibited a dose-dependent synergistic antifungal effect on the growth and biofilm formation of C. albicans isolates as determined by a XTT assay and fluorescent microscopy assay. The synergistic effect was accompanied with a significantly down-regulated expression of adhesion-related genes ALS3, hypha-related genes HWP1, ABC transporter drug-resistant genes CDR1 and MDR1, and FLC targeting gene, encoding sterol 14alpha-demethylase (ERG11) in clinical C. albicans isolates. Furthermore, an addition of CSA significantly reduced the cellular surface hydrophobicity but increased intracellular calcium concentration as determined by a flow cytometry assay $(p<0.05)$.

Conclusion: The results presented in this report demonstrated that the synergistic effect of CsA and FLC on inhibited C. albicans biofilm formation and enhanced susceptibility to FLC was in part through a mechanism involved in suppressing the expression of biofilm related and drug-resistant genes, and reducing cellular surface hydrophobicity, as well as evoking intracellular calcium concentration.
\end{abstract}

Keywords: Candida albicans, Biofilm, Calcineurin inhibitor, Cyclosporine A, Fluconazole

\section{Background}

The infection of Candida albicans (C. albicans) continues to be a major cause of high mortality among immunocompromised and hospitalized patients, and the bloodstream Candida infection has been listed as the third most common causes of nosocomial bacteremia and the most common etiologic agent of fungal-related biofilm infection $[1,2]$. With an ability to form biofilm seen in the most microorganisms, a formation of C. albican

\footnotetext{
* Correspondence: liuxiaoming@nxmu.edu.cn; weijun@nxmu.edu.cn 'Ningxia Key laboratory of Clinical and Pathogenic Microbiology, the General Hospital of Ningxia Medical University, Yinchuan, Ningxia 750004, China

Full list of author information is available at the end of the article
}

biofilm not only provides a protection from environmental stress, but it also allows a horizontal transfer of genes that potentially encode antibiotic resistance, sequentially enhances the resistance of microorganisms to an antimicrobial agent by up to 1000-fold greater than that needed for a treatment of their planktonic counterparts $[3,4]$.

Fluconazole (FLC) is a member of the azole class, organic compounds posses a five-membered heterocyclic ring with two double bonds, which is the most commonly used first-line agent in the prevention and treatment for patients with candidemia or suspected invasive candidiasis, through a mechanism by which the FLC is able to functionally target encoding sterol 14alpha-demethylase 
(ERG11), an essential enzyme in the ergosterol biosynthetic pathway of C. albican [5]. However, FLC was found to be ineffective in treatment of $C$. albicans biofilm, and the formation of biofilm has been demonstrated to contribute to the failure of anti-fungal treatment, including FLC and other agents, which has been attributed to a compromise in C. albicans cell membrane integrity caused by reduced sterols [6]. Intriguingly, mounting evidence has revealed that the antifungal activity of FLC in $C$. albicans biofilm killing could be synergistically enhanced when it was employed in a combination with some antibiotics or immunosuppressants [7-14]. Among them, the calcineurin inhibitors, such as cyclosporine A (CsA) and FK506 have spurred an increased interest [14-19].

Calcineurin is a $\mathrm{Ca}^{2+}$-calmodulin-activated phosphatase, which is a multifunctional regulator with functions in governing fungal stress responses, physiological and cell cycle progression, biofilm formation, antifungal resistance, virulence and pathogenesis, and is essential for C. albicans survival during membrane stress [20-23]. Several lines of evidence have uncovered that $C$. albicans was resistant to calcineurin inhibitors of CsA and FK506, despite some fungal species were susceptible to these agents. Notably, a combination of either CsA or FK506 with the fluconazole exhibited a synergistic anti-fungal activity to both of planktonic and biofilm C. albicans [14-17, 20, 24]. Particularly, the calcineurin inhibitor CsA was recently found to be able to enhance the susceptibility of biofilm-producing C. albicans to fluconazole [24]. These results implied that targeting calcineurin signaling using a combination of calcineurin inhibitor FK506 or CsA and FLC might be a promising antifungal strategy for prevention and treatment of biofilm $C$. albicans infection. However, the underlying mechanism by which a calcineurin inhibitor enhances the susceptibility of C. albicans to the most common antifungal agent, FLC has yet been fully understood.

In the present report, we aimed to interrogate the molecular mechanism of calcineurin inhibitor CsA in enhancing the susceptibility of biofilm-producing $C$. albicans to FLC by accessing its impacts on the alterations of the expression of drug-transporters and adhesion associated genes, cellular surface hydrophobicity $(\mathrm{CSH})$ and intracellular calcium $([\mathrm{Ca}(2+)])$ levels. Our results demonstrated that an addition of CsA led an enhanced susceptibility of $C$. albicans to FLC in part through a mechanism by down-regulating the expression of genes associated to $\mathrm{ABC}$ transporter and adhesion, a decrease of $\mathrm{CSH}$ and an increased intracellular calcium $([\mathrm{Ca}(2+)])$ level.

\section{Results}

\section{Biofilm-producing capacity of clinical Candida albicans} isolates

In order to evaluate the clinical significance of biofilm in clinical C. albicans infection, the capacity of biofilm formation of 24 C. albicans clinical isolates were examined. The result showed distinct biofilm-producing capacities of these clinical isolates, which could be categorized into three groups, strain with capacity of low biofilm formation (LBF), intermediate biofilm formation (IBF) and high biofilm formation (HBF), according the absorbance of $\mathrm{OD}_{590 \mathrm{~nm}}$ as described as previous report [25]. 6 clinical isolates were fell into $\mathrm{LBF}$ with an $\mathrm{OD}_{590 \mathrm{~nm}}$ value less than the first quartile $\left(\mathrm{Q} 1 \mathrm{OD}_{590 \mathrm{~nm}}=0.384\right), 12$ strains in $\mathrm{HBF}$ with an $\mathrm{OD}_{590 \mathrm{~nm}}$ value greater than the third quartile $(\mathrm{Q} 3$ $\left.\mathrm{OD}_{590 \mathrm{~nm}}=1.152\right)$, and 6 strains could be grouped in IBF with an $\mathrm{OD}_{590 \mathrm{~nm}}$ value between Q1 and Q2 in this report (Fig. 1a). Morphologically, patched biofilm with hyphal cells was formed in LBF strains (Fig. 1b), while an intact biofilms could be frequently observed in HBF isolates (Fig. 1c). This data indicated distinct biofilm-producing abilities among clinical $C$. albicans isolates. The isolate with the greatest capacity of biofilm formation (the greatest value of absorbance of $\mathrm{OD}_{590 \mathrm{~nm}}$ ) was selected for further investigation in this study (it was denoted as the red dot in Fig. 1a).

\section{CsA enhances the susceptibility of clinical biofilm- producing Candida albicans to fluconazole}

Calcineurin inhibitors, such as FK506 and CsA have been evidenced to enhance the susceptibility of $C$. albicans to azole agents $[14,17,18]$. In line with these findings, an addition of $75 \mu \mathrm{g} / \mathrm{mL}$ of CsA with FLC significantly led an enhanced susceptibility of clinical biofilm-producing $C$. albicans to FLC by 8- 32-fold over the FLC or CsA alone in the $6 \mathrm{HBF}$ isolates $(p<0.01)$ (Fig. 2a), although FLC alone also showed an insignificant inhibition $(<20 \%)$ of biofilm growth of C. albicans at concentration of $1024 \mu \mathrm{g} /$ $\mathrm{mL}$ (Fig. 2a). In addition, CsA alone also exhibited a moderate ability to inhibit of biofilm growth $(<10 \%)$ of $C$. albicans at concentration of $300 \mu \mathrm{g} / \mathrm{mL}$ (Data not shown). Morphological analysis using fluorescent microscopy further revealed that a combination of $32 \mu \mathrm{g} / \mathrm{mL}$ of FLC and $75 \mu \mathrm{g} / \mathrm{mL}$ of CsA was able to inhibit C. albicans cell growth and hyphal formation, while the cells could gradually mature to highly filamentous hyphal cells with a multi-dimensional structure when they were cultured in a naïve condition (Fig. $2 \mathrm{~b}, \mathrm{c}$ ).

\section{A combination of FLC and CsA alters C. albicans the expression of drug-resistant genes}

We next sought the potential molecular mechanism behind the ability of CsA to enhance the susceptibility of biofilm-producing $C$. albicans isolates to FLC, the expression of drug-resistant genes, such as agglutinin-like sequence 3 (ALS3), hyphal wall protein 1 (HWP1), candlda drug resistance 1 (CDR1), multidrug resistance 1 (MDR1) and ERG11 of cells exposed to $75 \mu \mathrm{g} / \mathrm{mL}$ CsA and $32 \mu \mathrm{g} / \mathrm{mL}$ FLC, was determined by a qRT-PCR assay. 


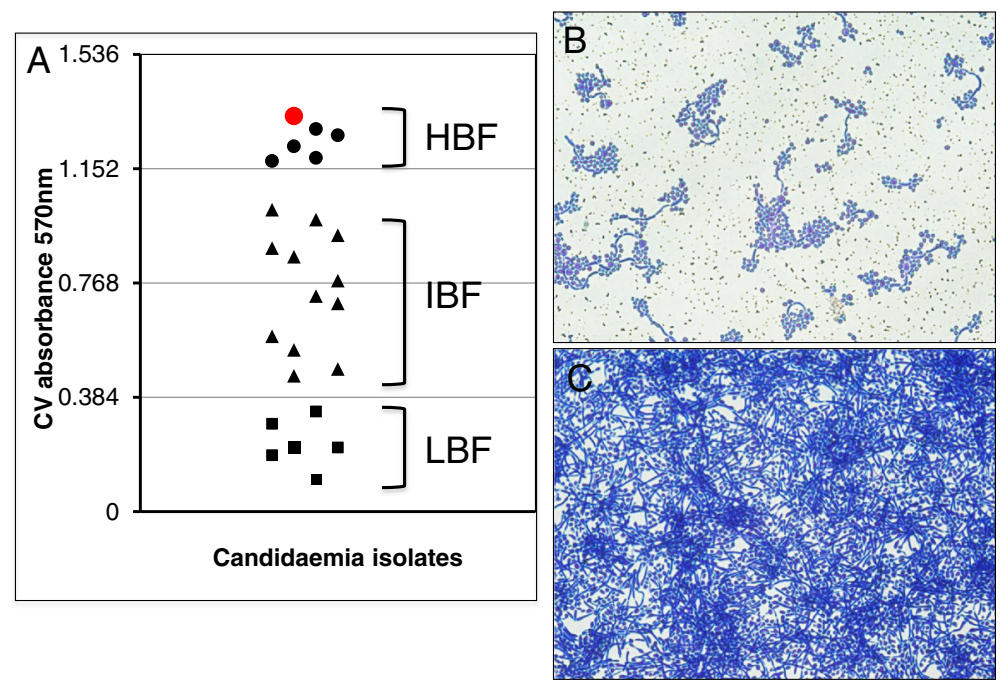

Fig. 1 Distinct biofilm forming capacity of clinical C. albicans strains isolated from bloodstream. Candida albicans isolates were cultured in a 6-well plates for $48 \mathrm{~h}$ to allow the maturation of biofilm. The biofilms were then stained with crystal violet, and visualized and imaged under a light microscope. A distinct capacity of biofilm formation was observed among the 24 clinical C. albicans isolates. a 24 clinical C. albicans isolates were categorized in LBF (low biofilm formation capacity) (filled squares), IBF (intermediate biofilm formation capacity) (filled triangles) and HBF (high biofilm formation capacity) (dots). The isolate marked with red dot in HBF group was chosen for further study in this report. b A representative image of biofilm produced by a LBF (low biofilm formation capacity) C. albicans isolate. c A representative images of biofilm produced by a HBF (high biofilm formation capacity) C. albicans isolate

Interestingly, a synergistically inhibitory effect on the expression of adhesion-related genes $A L S 3$, hypha-related genes HWP1, ABC transporter drug-resistant genes CDR1 and MDR1, and FLC targeting gene ERG11 was observed in the cells treated with a combination of CsA and FLC, which was statistically different as compared with those treated with these agents alone $(p<0.05$ or 0.01 ), albeit both CsA and FLC alone also displayed an ability to moderately suppress the expression of above tested gene in biofilm-producing $C$. albicans strains $(p<0.05$ or $p>0.05$ ) (Fig. 3). Of note, both CsA and FLC alone failed to inhibit the ERG11 gene expression in these clinical biofilmproducing C. albicans isolates (Fig. 3). This result imply that the CsA-induced synergistic effect on the enhanced susceptibility to FLC may be in part through a mechanism of down-regulation of the expression of genes associated with cell adhesion, hyphal formation and drug resistance.

\section{CsA and FLC synergistically reduce cellular surface hydrophobicity of the $C$. albicans strain}

A compelling body of studies has demonstrated that the cellular surface hydrophobicity (CSH) is positively correlated with the adhesion and morphological transition, and key processes of C. albicans biofilm formation [26-28]. In order to unravel whether a combination of CsA and FLC has an impact on the biofilm formation of C. albicans, the $\mathrm{CSH}$ of the fungi cells with different treatments was examined. Despite the fungi cells exposed to both CsA and FLC alone showed a significantly decreased CSH relative to the untreated controls $(p<0.05)$, while a combination of CsA and FLC further dramatically inhibited CSH in comparison with the untreated controls $(p<0.01)$ and the cells treated with CsA or FLC alone $(p<0.05)$ (Fig. 4). The evidence suggests that a combination of CsA and FLC has a synergistic effect on the reduction of cellular surface hydrophobicity in these clinical C. albicans isolates.

\section{CsA and FLC synergistically increases intracellular calcium in C. albicans}

Since the calcium homeostasis is essential in developmental and stress signaling pathways in C. albicans [29], the calcium-associated pathways have important implications in key pathogenic steps of this fungal species [30]. Given the fact of that the calcineurin was an important regulator of $\mathrm{Ca}^{2+}$ pathways [21], we thus next investigated whether the synergistic antifungal effect of CsA and FLC was associated with calcium homeostasis disturbance, as seen in a previously demonstrated in a combination of minocycline and fluconazole and caused a significant increase of intracellular calcium [13]. As expected, a combination of calcineurin inhibitor CsA and FLC indeed induced a time dependent increase of intracellular calcium, such a fluctuation of $\mathrm{Ca}^{2+}$ level was statistically different in comparison with these agents alone, as determined by a FACS assay using Fluo-3/AM indicator staining $(p<0.05$ for $6 \mathrm{~h}$ of treatment, and $p<0.01$ for $12 \mathrm{~h}$ of challenge) (Fig. 5). Intriguingly, the clinical $C$. albicans cells exposed to either CsA or FLC alone exhibited rather lower intracellular levels of $\mathrm{Ca}^{2+}$ with slightly changes over time after a $6 \mathrm{~h}$ of treatments relative to untreated controls $(p>0.05)$ (Fig. 5). 

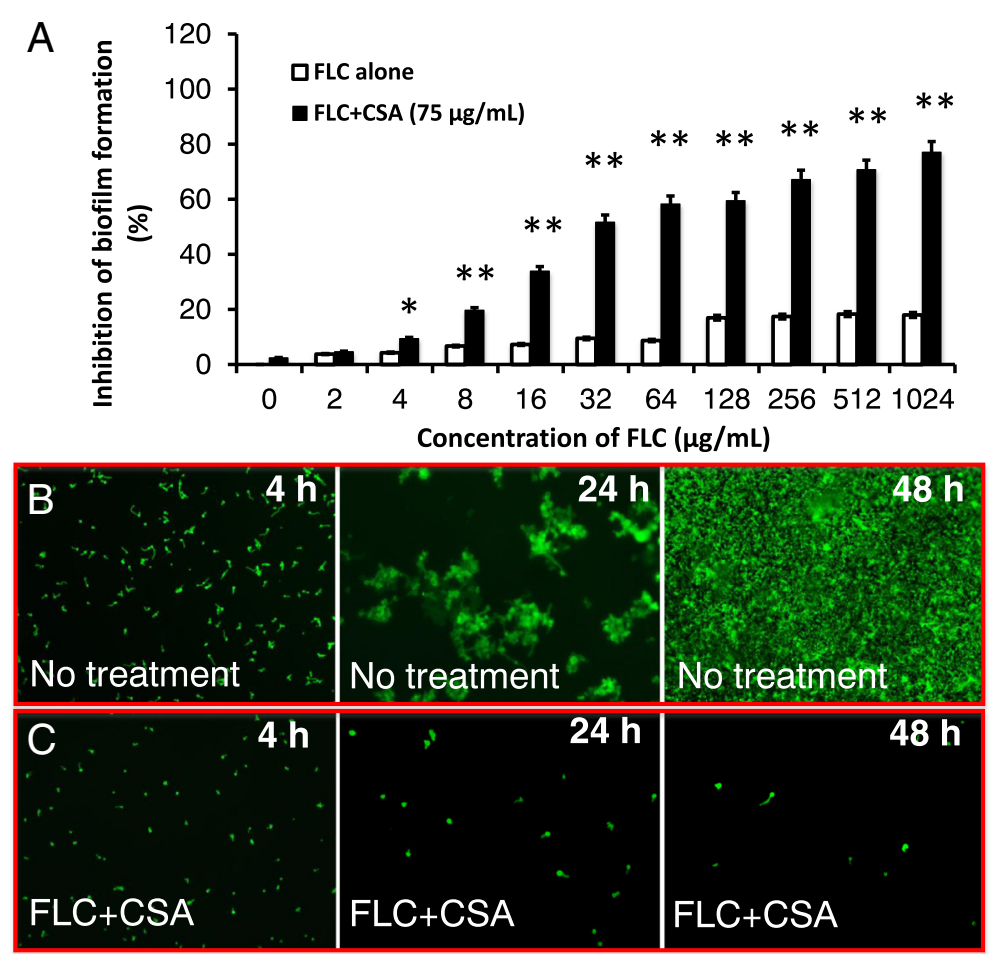

Fig. 2 A synergistic effect of calcineurin inhibitor cyclosporine A (CSA) on increasing susceptibility of C. albicans isolates to fluconazole. C. albicans isolates were cultured in 96-well plates with indicated concentration of FLC or a combination of the indicated concentration of FLC and $75 \mu \mathrm{g} /$ $\mathrm{mL}$ of CsA for $24 \mathrm{~h}$ or $48 \mathrm{~h}$. The inhibition of fungi growth was accessed in terms of a XTT assay and fluorescent microscopy. a CsA significantly enhances FLC-induced inhibition of the growth of C. albicans biofilm-producing isolates at $24 \mathrm{~h}$ post-incubation, as compared with FLC alone. Compared with FLC alone group, ${ }^{*}: p<0.05 ;{ }^{* *}: p<0.01$. Data in A represented the mean $\pm \mathrm{SD}$ from three independent triplicated experiments $(N=9)$. b , c. Representative images of biofilm formation of a HLF C. albicans isolate for culturing at indicated time, as determined by a fluorescent microscopy assay. b. Untreated group. c. FLC+CsA treated group. In comparison with the untreated group (b), a combination of $75 \mu \mathrm{g} / \mathrm{mL}$ of CsA and $32 \mathrm{\mu g} / \mathrm{mL}$ of FLC dramatically inhibited the cell growth and biofilm formation (c)

This data clearly indicated that the combination of calcineurin inhibitor CsA and the most commonly used antifungal agent FLC could inhibit the growth of biofilmproducing $C$. albicans clinical isolates by disturbing their intracellular calcium homeostasis.

\section{Discussion}

Candida albicans is an important nosocomial infectious agent, and an infection of biofilm-producing Candida albicans among immunocompromised patients remains a clinical challenge. In this regard, the use of medical devices such as central venous catheters (CVC's) and prostheses is a well-known risk factor to induce biofilm formation [31, 32]. Despite a significant advance in our knowledge such as the molecular mechanism of $C$. albicans biofilm formation has been made over the past decade, there is no ideal therapeutic method for bloodstream infections caused by biofilm-producing Candida albicans in clinical practice [33].

Accumulating evidences have revealed that a formation of biofilm of $C$. albicans could enhance the resistance of this fungi species to most of the commonly used antifungal agents $[1,34,35]$. Therefore, it is urgent to discover novel antifungal agents or regimens based on new drug targets for the treatment of bloodstream infections, particularly an infection of MDR-biofilm-producing C. albicans. With this respect, several lines of evidence have uncovered that a combination of calcineurin inhibitor, such as FK506 and CsA, or antibiotics could synergistically enhance the susceptibility of biofilm-producing C. albicans to the firstline antifungal agent FLC $[8,10-14,17,18]$. In the present report, we also demonstrated that the calcineurin inhibitor CsA had a potential to increase the susceptibility of clinical biofilm-producing $C$. albicans to FLC by suppressing their abilities to form biofilm, and inhibiting the expression of genes related to cell adhesion, hyphal formation and drugtransportators, as well as decreasing cellular surface hydrophobicity and increasing intracellular calcium concentration.

Previous studies have reported that biofilms formed by C. albicans strains that isolated from bloodstreams displayed phenotypes associated with drug-resistance and pathogenicity [25]. Therefore, we aimed to morphologically assess biomasses of $C$. albicans clinical isolates using 


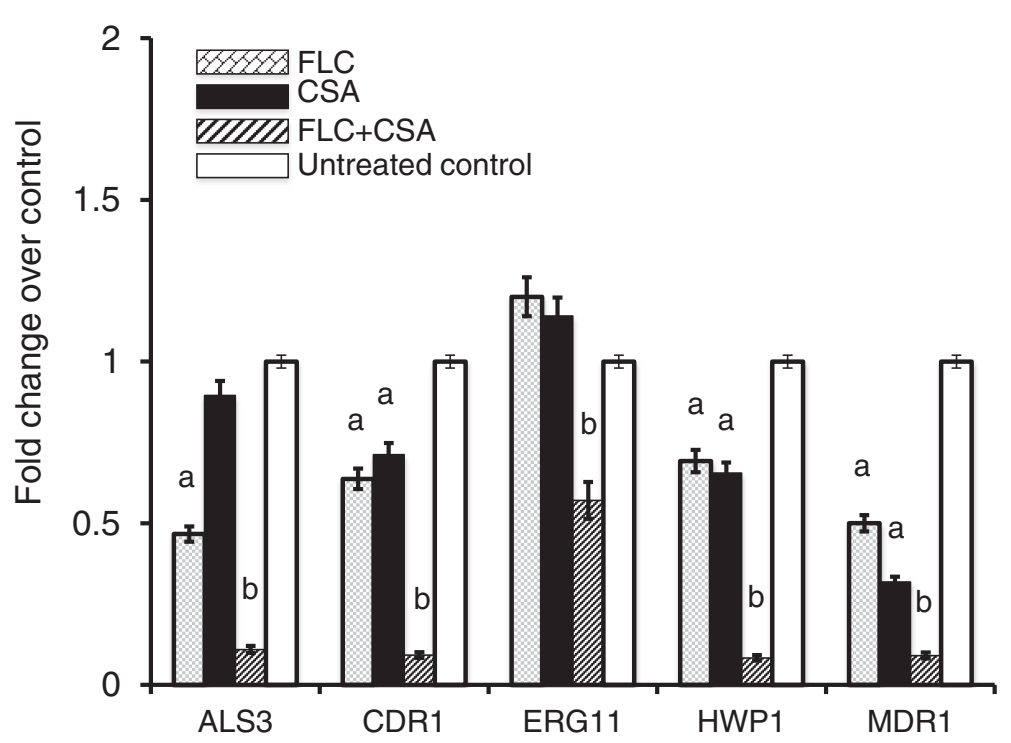

Fig. 3 A combination of calcineurin inhibitor CSA and FLC down-regulated the expression of biofilm-related and drug transporter in clinical C. albicans isolates. Clinical C. albicans isolates were cultured in 6-well plates with $32 \mu \mathrm{g} / \mathrm{mL}$ FLC and $75 \mu \mathrm{g} / \mathrm{mL}$ of CsA alone or in combination for $24 \mathrm{~h}$. The expression of indicated genes was determined by a qRT-PCR assay. The combination of CSA and FLC significantly inhibited the expression of all tested genes, although CSA or FLC along also could moderately down-regulated the expression of these genes. Compared with the untreated group, FLC alone group, a: $p<0.05$; compared with the CSA or FLC alone group, b: $p<0.01$. Data represented the mean \pm SD from three independent triplicated experiments $(N=9)$

the crystal violet staining, and found distinct capacities of biofilm formations of clinical Candida albicans bloodstream isolates, suggesting that the biofilm producing capacity may have an implication of clinical significance. Interestingly, an addition of CsA was verified to be able synergistically increase the susceptibility of these isolates to

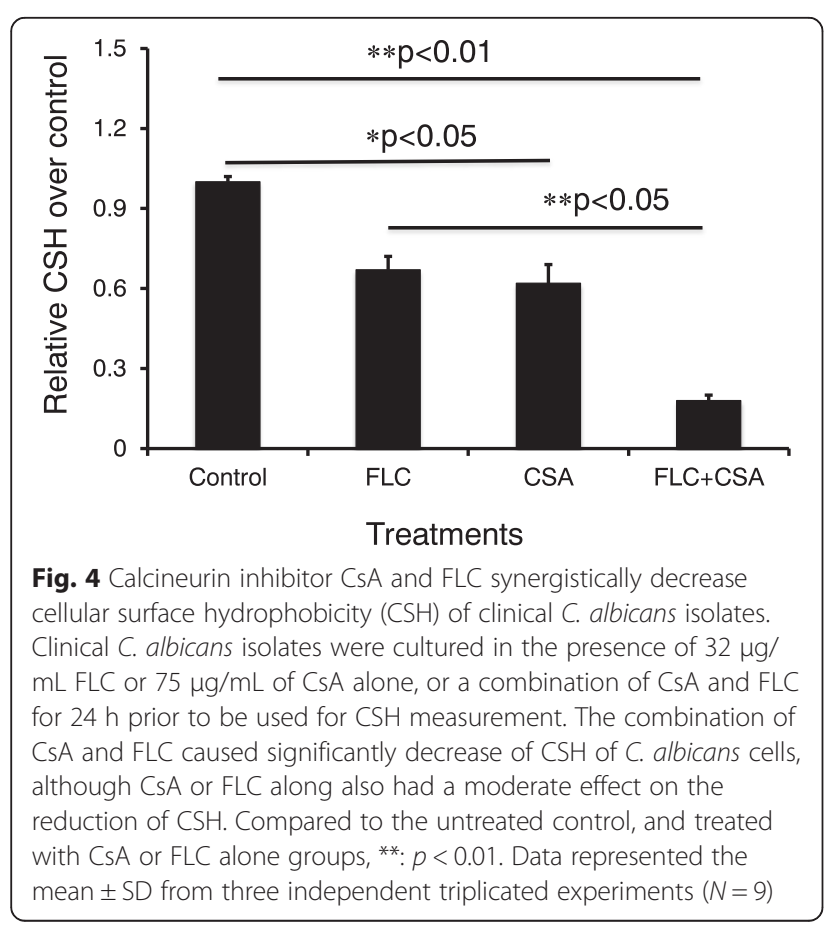

FLC, along with a down-regulation of the expression of ALS3, HWP1, CDR1, MDR, ERG11 genes. Among them, $A L S 3$ are members of the agglutinin-like sequence (ALS) gene family that encodes cell-wall glycoproteins [36], and both $A L S$ and HWP1 genes are highly expressed in hyphae and play essential roles in the yeast-to-hypha morphological transition of C. albicans, in which the ALS3 contributes cell adhesions, and HWP1 mediates cell substrate and cell-cell interactions in biofilms [37-39]. Therefore, a combination of CsA and FLC-induced down-regulation of these genes might contribute to the anti-biofilm effect by targeting the three known stages for biofilm formation: adhesion to biomaterial surfaces, growth to form an anchoring layer, and morphological transition to form a complex threedimensional structure $[40,41]$. Of note, no alteration or marginal changes of the expression of these genes was found in cells treated with CsA and FLC alone in this study, indicating that the CsA or FLC had limited effect on biofilm growth of C. albicans. Equally noteworthy, FLC alone exhibited a limited effect on ERG11 gene expression, which may be in part due to that HBF isolates were more resistant to FLC than LBF strains, and more abundant ERG11 transcripts were to reported to be detected in FLCresistant CA strains [42].

The azoles are generally fungistatic agents for treatment and prevention of C. albicans infections [43]. However, azole resistant biofilm-producing $C$. albicans infections were frequently observed in clinic settings, which have been attributed to interactions of multiple mechanisms 

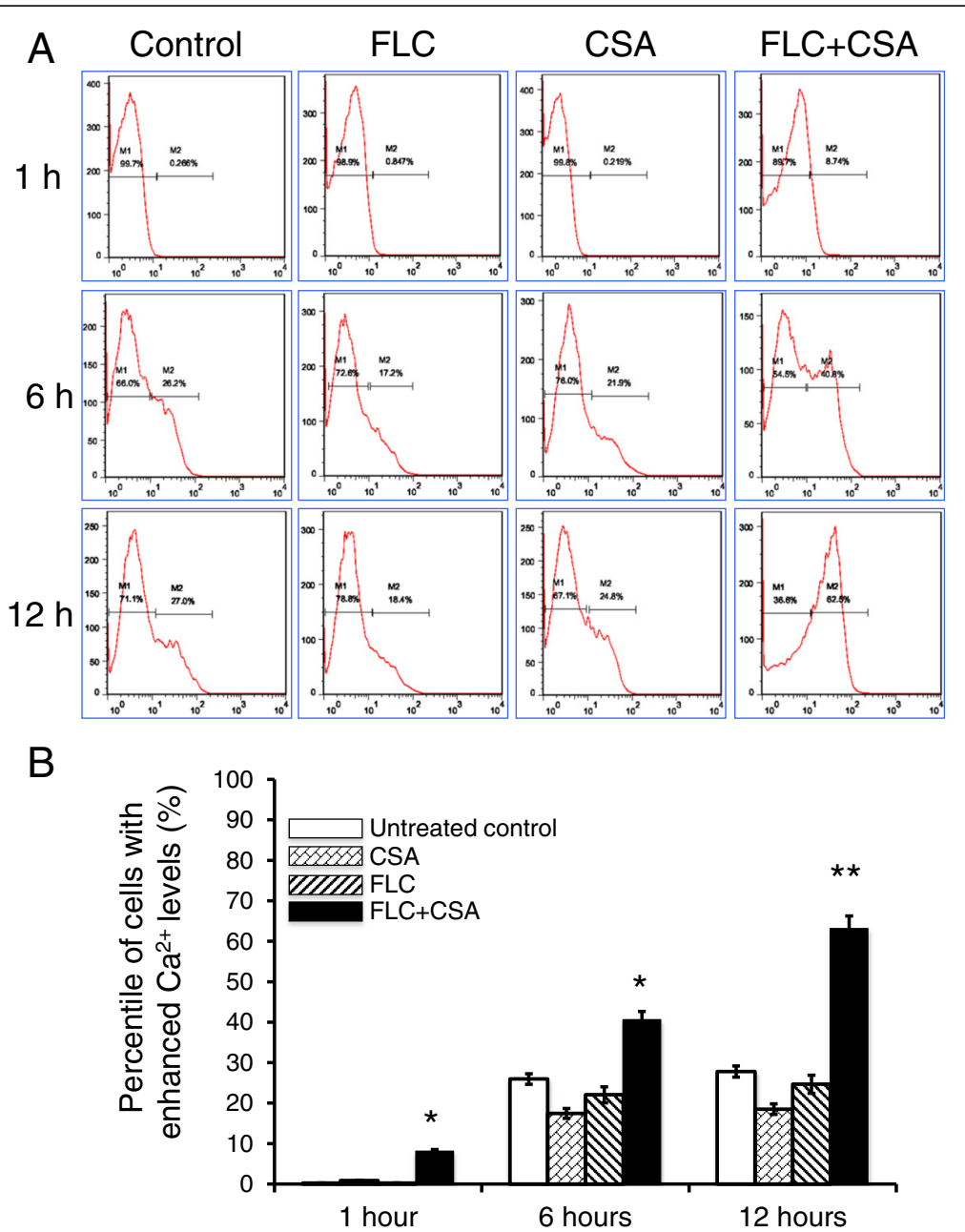

Fig. 5 Calcineurin inhibitor CSA and FLC synergistically increase intracellular calcium concentration clinical C. albicans isolates. Clinical C. albicans isolates were exposed to $32 \mu \mathrm{g} / \mathrm{mL}$ FLC or $75 \mu \mathrm{g} / \mathrm{mL}$ of CsA alone, or a combination of CsA and FLC for $24 \mathrm{~h}$ prior to be used for determining intracellular calcium concentration by a flow cytometric assay (FACS). a Representative plot images of FACS analysis showed the fraction of cells with high intracellular calcium concentration (M2 fraction) treated with indicated conditions. $\mathbf{b}$ Quantitative analysis of percentages of cells with high intracellular calcium levels. The result showed that both CSA and FLC alone could reduce intracellular calcium, but a combination of them caused a time-dependently evoked intracellular calcium concentration. Compared with the untreated group of each indicated time point, *: $p<0.05 ;{ }^{* *}: p<0.01$. Data represented the mean \pm SD from three independent triplicated experiments $(N=9)$

including the alteration of ERG11 gene expression [44]. ERG11 gene encodes the $14 \alpha$-demethylase enzyme which has an effect on ergosterol biosynthesis, and an upregulated expression of this gene in biofilm $C$. albicans isolates may explain their resistance to azole [45]. In agreement with this notion, exposing biofilm-producing $C$. albicans isolates to a combination of CsA and FLC caused adown-regulation of ergosterol biosynthesis-related gene ERG11, which implied an underlying mechanism by which calcineurin inhibitors have potentials to enhance the susceptibility of biofilm-producing C. albicans to FLC [14]. It has been previously demonstrated that the highly frequent azole resistance in C.albicans strains was in part attributed to an increased efflux of drug mediated mostly by the ATP-binding cassette $(\mathrm{ABC})$ and the major facilitator superfamily (MFS) transporters [46, 47]. In this context, the expression of genes encoding both types of efflux pumps was up-regulated during the course of biofilm formation and development in C. albicans [47]. Controversially, a later study by Marchetti et al. suggested that a synergistic antifungal effect of cyclosporine and FLC in C. albicans was multidrug efflux transporter genes CDR1, CDR2, MDR1 and FLU1 independent [48]. Inconsistent with this finding, we found that there was a significant down-regulation of efflux transporter genes CDR1 and MDR1 in clinical biofilm-producing $C$. albicans isolates treated with a combination of CsA and FLC, suggesting that the CsAmediated increase of susceptibility of biofilm-producing $C$. albicans to FLC is at least in part through a mechanism by suppressing the expression of these drug transporter genes. 
The cell surface hydrophobicity (CSH) of Candida species has an implication in the adhesion and biofilm formation of the organisms on epithelial cells or medical device $[26,49]$, which is also associated with the fungicidal resistance [50-52]. For instance, a FLC resistant C. tropicalis strain exhibited a significantly more hydrophobic, greater adherence and higher capacity of biofilm formation on polystyrene surface relative to its parent strain that susceptible to FLC, along with an increased expression of $M D R 1$ and ERG11 genes and enhanced virulence in mice [50]. The discrepancy of CSH and biofilm formation capacity between FLC-susceptible and resistant strains was also recently reported in C. albicans cells cultured with different media in presence or absence of FLC [51]. In this regard, $C$. albicans cells dispersed from mature biofilms were more hydrophobic than those dispersed from earlier development stages of biofilms, and C. albicans isolates with high capacity of biofilm formation displayed an increased CSH relative to those with lower biofilm formation potential [51]. In agreement with these findings, our result also indicated that calcineurin inhibitor CsA could enhance susceptibility of biofilm-producing C. albicans isolates to FLC and prevent cell adhesion on polystyrene surface and biofilm formation (with $\mathrm{CSH}$ as the indicator) in part by decreasing $\mathrm{CSH}$.

$\mathrm{Ca}^{2+}$ burst is a common cellular response of $C$. albicans cells in response to an environmental stress [53]. It is often along with an activation of calcineurin signaling pathways, in which the calcineurin is required for survival in serum, virulence, and resistance to azole antifungals, in part via its downstream target, Crz1 transcription factor [53-56]. In the present study, a significant $\mathrm{Ca}^{2+}$ burst was observed in cells exposed to the combination of CsA and FLC. Of note, either FLC or CsA alone showed an ability to decrease intracellular calcium concentration, however a combination of these two agents had a synergistic effect on increase but not decrease of intracellular calcium $\left(\left[\mathrm{Ca}^{(2+)}\right]\right)$ levels. Such CsA-evoked intracellular calcium concentration might disturbed the calcium homeostasis and influenced the cell survival, which may partially explain the potential of CsA to enhance the effectiveness of FLC against the clinical biofilm-producing C. albicans. In addition, intracellular calcium was related to biofilm formation. For example, in a study on plant-pathogenic bacterium, Xylella fastidiosa, Cruz et al. demonstrated that intracellular calcium played a role in biofilm formation, which was related to the initial surface and cell-tocell attachment and colonization stages of biofilm establishment, and was depended on functions of fimbrial structures [57].

\section{Conclusion}

In the present study, we provided additional evidences that calcineurin inhibitors (such as CsA) were able to enhance the susceptibility of $C$. albicans clinical biofilm- producing isolates to the most commonly used fungicidal agent, fluconazole (FLC). Mechanistically, CsA could synergistically suppress the expression of adhesion-related genes $A L S 3$, hypha-related genes $H W P 1, \mathrm{ABC}$ transporter drugresistant genes $C D R 1$ and $M D R 1$, and FLC targeting gene ERG11 in biofilm producing C. albicans. In addition, a combination of CsA and FLC also could synergistically reduce cellular surface hydrophobicity $(\mathrm{CSH})$ and increase intracellular calcium concentration in biofilm-producing $C$ albicans isolates. Together with other studies, these results clearly suggest a combination of calcineurin inhibitor and fluconazole may prove to be a novel and effective therapeutic option, which warrants for further investigation.

\section{Methods}

Candida albicans strains and culture and identification Candida albicans 1strain SC5314 was purchased from American Type Culture Collection (Mannasas, VA, USA). 24. FLC sensitive $C$. albicans clinical strains were isolated from bloodstream samples and collected from the department of laboratory medicine of the General Hospital of Ningxia Medical University between September 2014 and January 2015, which were identified by harnessing the VITEK-2 COMPACT fully automated microbiological system. The $C$. albicans strains were routinely grown in YPD liquid medium (20 g of glucose per liter, $10 \mathrm{~g}$ of yeast extract, $20 \mathrm{~g}$ of peptone) at $30{ }^{\circ} \mathrm{C}$ with $5 \% \mathrm{CO}_{2}$ atmosphere [58]. All strains had normal and comparable growth rates. Human blood samples were collected with a protocol approved by the Ethic Committee for the Conduct of Human Research at Ningxia Medical University (NXMU2016-092). Written consent was obtained from every individual according to the Ethic Committee for the Conduct of Human Research protocol.

\section{Characterization of Candida albicans biofilm formation}

Candida albicans cells were grown in YPD overnight at $37{ }^{\circ} \mathrm{C}$ and resuspended in RPMI buffered with HEPES at a concentration of $1.0 \times 10^{6}$ cells $/ \mathrm{mL}$ prior to be applied for biofilm formation culturing. The biofilm model was established using a method described in a previous study [59]. Briefly, an $100 \mu \mathrm{L}$ of above cell suspension was seeded in a flat-bottomed 96 well plates with and incubated at $37{ }^{\circ} \mathrm{C}$ at with $5 \% \mathrm{CO}_{2}$ atmosphere for $24 \mathrm{~h}$ or until formation of mature biofilms, and biomass of each isolate was assessed in terms of the crystal violet (cv) assay by determining the distribution of biomass using the value of $\mathrm{OD}_{570 \mathrm{~nm}}$ as previously reported [60]. A $C$. albicans isolate with a less than the 1st quartile (Q1) was grouped as having low biofilm formation (LBF) capacity, and a isolate with a biomass greater than the 3rd quartile (Q3) was considered isolates with high biofilm formation (HBF) ability, and an isolate that lay in between Q1 and Q2 was a deemed strain with intermediate 
biofilm formation (IBF Q2) potency (Fig. 1a) [25]. After the culturing or treatment, harvested the cells by washing and scratching off from the culture wells, the cell suspension was then centrifuged for harvesting cell pellet.

\section{Test of antifungal susceptibility of biofilm-producing $C$.} albicans isolates

The antifungal susceptibility of biofilm-producing C. albicans was ascertained by determine minimum inhibitory concentration (MIC) of fungal cells on $24 \mathrm{~h}$ preformed biofilms, as previously described in flat-bottomed, 96 well microtitre plates [60]. The MIC was determined at $80 \%$ inhibition of fungal cells using an XTT (2,3-bis(2-methoxy-4nitro-5-sulfo-phenyl)-2H-tetrazolium-5-caboxanilide) metabolic reduction assay $[61,62]$. The tested range of concentrations of agents was $2 \mu \mathrm{g} / \mathrm{mL}$ to $1024 \mu \mathrm{g} / \mathrm{mL}$ for FLC, and $9.3 \mu \mathrm{g} / \mathrm{mL}$ to $300 \mu \mathrm{g} / \mathrm{mL}$ for CsA. Combinations of these two agents were prepared in a chequerboard format as previously reported [63]. All C. albicans strains were tested in duplicate for three independent experiments.

\section{Fluorescence microscope assay}

In order to morphologically observe the formation and integrity of $C$. albicans biofilm, biofilms cultured under different conditions were stained with $50 \mu \mathrm{g} / \mathrm{mL}$ FITCconA, and imaged using a fluorescent microscopy.

\section{Quantitative reverse transcriptional PCR (qRT-PCR)}

Candida albicans cells were homogenized using liquid nitrogen grinding method, and the total RNA was extracted using an RNA purification kit (TaKaRa Biotechnology, Dalian, China). The first strand of cDNA was synthesized by reverse transcription using a commercial RT kit (TaKaRa Biotechnology, Dalian, China). The thermal cycling condition was $94{ }^{\circ} \mathrm{C}$ for $4 \mathrm{~min}$ as an initial denaturation step, followed by 37 cycles of PCR, consisting of $94{ }^{\circ} \mathrm{C}$ for $30 \mathrm{~s}, 57^{\circ} \mathrm{C}$ for $30 \mathrm{~s}$ and $72{ }^{\circ} \mathrm{C}$ for $30 \mathrm{~s}$. After reacting, a melting curve was evaluated the specificity of the primers. The primer sets for amplifying genes of agglutinin-like sequence 3 (ALS3), hyphal wall protein 1 (HWP1), candlda drug resistance 1 (CDR1), multidrug resistance 1 (MDR1) and ERG11 were listed in Table 1 [64]. The result was analyzed using $2^{-(\Delta \Delta \mathrm{Ct})}$ [65]. The gene of $18 \mathrm{~S}$ rRNA was used as an endogenous reference control for normalization the relative expression, and the data was interpreted as fold of changes over the untreated controls. All analysis was carried out on data from three independent experiments with three replicates.

\section{Cellular surface hydrophobicity assay}

Since a hyphal form of $C$. albicans showed higher affinity for hydrocarbon than the yeast form, and the adherence of these fungus to hydrophobic surfaces increased when its morphology was changed from the yeast form
Table 1 Primer sequences used in this study

\begin{tabular}{lll}
\hline Gene & Primer sequences (5'-3') & Length \\
\hline CDR1-F & ACTCCTGCTACCGTGTTGATTG & 192 \\
$R$ & ACCTGGACCACTTGGAACATATTG & \\
ERG11-F & AAGAATCCCTGAAACCAA & 134 \\
R & CAGCAGCAGTATCCCATC & \\
MDR-F & GGTGCTGCTACTACTGCTTCTG & 226 \\
R & TGATGAAACCCAACACGGAACTAC & \\
HWP1-F & GCTCAACTTATTGCTATCGCTTATTACA & 105 \\
R & GACCGTCTACCTGTGGGACAGT & \\
ALS3 -F & CAACTTGGGTTATTGAAACAAAAACA & 80 \\
R & AGAAACAGAAACCCAAGAACAACCT & \\
$18 S$ rRNA-F & GGATTAACTGAAGACTAACTACTG & 144 \\
R & GAACAACAACCGATCCCTAGT & \\
\hline
\end{tabular}

to the hyphal form [66], C. albicans cellular surface hydrophobicity $(\mathrm{CSH})$ was assessed using a water-hydrocarbon two-phase assay as described previously [52]. Briefly, $C$. albicans isolates were standardized to $1 \times 10^{6}$ cells $/ \mathrm{mL}$ in RPMI-1640 and $24 \mathrm{~h}$ at $37^{\circ} \mathrm{C}$ and washed twice with PBS. C. albicans biofilms were scraped off to obtain a cell suspension $\left(\mathrm{OD}_{600 \mathrm{~nm}}, 1.0 \mathrm{~mL}\right.$ in YPD medium). Then, $1.2 \mathrm{~mL}$ of cell suspension was transferred into a clean glass tube for each group and overlayed with $0.3 \mathrm{~mL}$ of octane. The cell suspension was incubated at $30{ }^{\circ} \mathrm{C}$ for $10 \mathrm{~min}$ prior the aqueous phase to be measured $\mathrm{OD}_{600 \mathrm{~nm}}$ [25]. CSH was calculated using a formula as $\left(\left[\mathrm{OD}_{600 \mathrm{~nm}}\right.\right.$ of control - OD6 $6_{00 \mathrm{~nm}}$ of test]/OD600nm of control) $\times 100 \%$ as previously described [58].

\section{Detection of intracellular calcium $\left(\left[\mathrm{Ca}^{(2+)}\right]\right)$ level}

Candida albicans biofilms with different treatments were stained with $5 \mu \mathrm{mol} / \mathrm{L}$ of calcium-sensitive indicator Fluo3/AM (Invitrogen, USA) in light proof at $37{ }^{\circ} \mathrm{C}$ for $30 \mathrm{~min}$. The cells were then washed three times with DHanks buffer (Invitrogen, USA). The calcium levels were determined by flow cytometry in a FACScan flow cytometer (Becton Dickinson, USA) using a parameter of the excitation/emission wave lengths $(485 \mathrm{~nm} / 530 \mathrm{~nm})$ with reading sensitivity level at 8 [10].

\section{Statistical analysis}

All data were recorded and analyzed by using the WHONET software (version 5.6). The statistical analysis was processed with the Statistical Package for the Social Sciences (SPSS) software (SPSS, version 18.0, Chicago, IL, USA). The changes of MICs for MDR C. albicans isolates between FLC or CsA alone and a combination of them were compared with a $t$-test analysis. Data were represented as the mean $\pm \mathrm{SD}$. A $p<0.05$ was defined as a statistical significance. 


\section{Abbreviations}

ABC, ATP-binding cassette; ALS3, agglutinin-like sequence 3; C. albicans: Candida albicans; CDR1, candlda drug resistance 1; CSA, cyclosporine A; CSH, cellular surface hydrophobicity; CVC, central venous catheters; ERG11, encoding sterol 14alpha-demethylase; FLC, fluconazole; HBF, high biofilm formation; HWP1, hyphal wall protein 1; IBF, intermediate biofilm formation; LBF, low biofilm formation; MDR1, multidrug resistance $1 ; X T T$, 2,3-Bis-(2-methoxy-4-nitro-5-sulfophenyl) -2H-tetrazolium-5-carboxanilide

\section{Acknowledgements}

The authors thank Ms. Yuying Zhang, Mr. Fei Han, Mr. Anquan Shang and Mr. Jiali Yang for their valuable discussion and assistance.

\section{Funding}

This work was supported by competing grants from the Ningxia Key Laboratory of Clinical and Pathogenic Microbiology for WJ and JW (LCPM201501-I and LCPM201502-I). The funders had no role in study design, data collection and analysis, decision to publish, or preparation of the manuscript.

\section{Availability of data and materials}

All the data supporting our findings is contained within the manuscript.

\section{Authors' contributions}

WJ, XL and JW conceived and designed the experiments; JW, HZ, CY and GL analyzed the data and drafted the manuscript; $\mathrm{HZ}, \mathrm{CY}$ and $\mathrm{GL}$ performed experiments and acquired data; WJ, HZ and GL collected samples; JW and XL interpreted data and critically revised the manuscript. All authors read and approved the final version of the manuscript.

\section{Competing interests}

The authors declare that they have no competing interests.

\section{Consent for publication}

Not applicable

\section{Ethics approval and consent to participate}

Human blood samples were collected with a protocol approved by the Ethic Committee for the Conduct of Human Research at Ningxia Medical University (NXMU-2016-092). Written consent was obtained from every individual according to the Ethic Committee for the Conduct of Human Research protocol.

\section{Author details \\ 'Ningxia Key laboratory of Clinical and Pathogenic Microbiology, the General Hospital of Ningxia Medical University, Yinchuan, Ningxia 750004, China. ${ }^{2}$ Center of Laboratory Medicine, the General Hospital of Ningxia Medical University, Yinchuan, Ningxia 750004, China. ${ }^{3}$ The First People's Hospital of Mudanjiang City, Mudanjiang, Helongjiang 157011, China. ${ }^{4}$ Department of Laboratory Medicine, College of Clinical Medicine, Ningxia Medical University, Yinchuan, Ningxia 750004, China.}

\section{Received: 8 December 2015 Accepted: 6 June 2016} Published online: 18 June 2016

\section{References}

1. Ramage G, Rajendran R, Sherry L, Williams C. Fungal biofilm resistance. Intern J Microbiol. 2012;2012:528521.

2. Sun L, Liao K, Wang D. Effects of magnolol and honokiol on adhesion, yeast-hyphal transition, and formation of biofilm by Candida albicans. PLoS One. 2015;10(2), e0117695.

3. Zhang L, Keogh S, Rickard CM. Reducing the risk of infection associated with vascular access devices through nanotechnology: a perspective. Int J Nanomedicine. 2013:8:4453-66.

4. Akbari F, Kjellerup BV. Elimination of bloodstream infections associated with Candida albicans biofilm in intravascular catheters. Pathogens. 2015:4(3):457-69.

5. Vanden Bossche $\mathrm{H}$. Biochemical targets for antifungal azole derivatives: hypothesis on the mode of action. Curr Top Med Mycol. 1985;1:313-51.

6. Mukherjee PK, Chandra J, Kuhn DM, Ghannoum MA. Mechanism of fluconazole resistance in Candida albicans biofilms: phase-specific role of efflux pumps and membrane sterols. Infect Immun. 2003;71(8):4333-40.
7. Chen YL, Lehman VN, Averette AF, Perfect JR, Heitman J. Posaconazole exhibits in vitro and in vivo synergistic antifungal activity with caspofungin or FK506 against Candida albicans. PLoS One. 2013;8(3), e57672.

8. Fiori A, Van Dijck P. Potent synergistic effect of doxycycline with fluconazole against Candida albicans is mediated by interference with iron homeostasis. Antimicrob Agents Chemother. 2012;56(7):3785-96.

9. Gamarra S, Rocha EM, Zhang YQ, Park S, Rao R, Perlin DS. Mechanism of the synergistic effect of amiodarone and fluconazole in Candida albicans. Antimicrob Agents Chemother. 2010;54(5):1753-61.

10. Gao Y, Li H, Liu S, Zhang X, Sun S. Synergistic effect of fluconazole and doxycycline against Candida albicans biofilms resulting from calcium fluctuation and downregulation of fluconazole-inducible efflux pump gene overexpression. J Med Microbiol. 2014:63(Pt 7):956-61.

11. Gao Y, Zhang C, Lu C, Liu P, Li Y, Li H, Sun S. Synergistic effect of doxycycline and fluconazole against Candida albicans biofilms and the impact of calcium channel blockers. FEMS Yeast Res. 2013;13(5):453-62.

12. Sasaki E, Maesaki S, Miyazaki Y, Yanagihara K, Tomono K, Tashiro T, Kohno S. Synergistic effect of ofloxacin and fluconazole against azole-resistant Candida albicans. J Infect Chemother. 2000;6(3):151-4.

13. Shi W, Chen Z, Chen X, Cao L, Liu P, Sun S. The combination of minocycline and fluconazole causes synergistic growth inhibition against Candida albicans: an in vitro interaction of antifungal and antibacterial agents. FEMS Yeast Res. 2010;10(7):885-93.

14. Uppuluri P, Nett J, Heitman J, Andes D. Synergistic effect of calcineurin inhibitors and fluconazole against Candida albicans biofilms. Antimicrob Agents Chemother. 2008;52(3):1127-32.

15. Onyewu C, Afshari NA, Heitman J. Calcineurin promotes infection of the cornea by Candida albicans and can be targeted to enhance fluconazole therapy. Antimicrob Agents Chemother. 2006;50(11):3963-5.

16. Onyewu C, Blankenship JR, Del Poeta M, Heitman J. Ergosterol biosynthesis inhibitors become fungicidal when combined with calcineurin inhibitors against Candida albicans, Candida glabrata, and Candida krusei. Antimicrob Agents Chemother. 2003;47(3):956-64

17. Reedy JL, Husain S, Ison M, Pruett TL, Singh N, Heitman J. Immunotherapy with tacrolimus (FK506) does not select for resistance to calcineurin inhibitors in Candida albicans isolates from liver transplant patients. Antimicrob Agents Chemother. 2006;50(4):1573-7.

18. Steinbach WJ, Reedy JL, Cramer Jr RA, Perfect JR, Heitman J. Harnessing calcineurin as a novel anti-infective agent against invasive fungal infections. Nat Rev Microbiol. 2007:5(6):418-30.

19. Zhang J, Silao FG, Bigol UG, Bungay AA, Nicolas MG, Heitman J, Chen YL. Calcineurin is required for pseudohyphal growth, virulence, and drug resistance in Candida lusitaniae. PLoS One. 2012;7(8), e44192.

20. Cruz MC, Goldstein AL, Blankenship JR, Del Poeta M, Davis D, Cardenas ME, Perfect JR, McCusker JH, Heitman J. Calcineurin is essential for survival during membrane stress in Candida albicans. EMBO J. 2002;21(4):546-59.

21. Moser MJ, Geiser JR, Davis TN. Ca2 + -calmodulin promotes survival of pheromone-induced growth arrest by activation of calcineurin and Ca2 + -calmodulin-dependent protein kinase. Mol Cell Biol. 1996:16(9):4824-31.

22. Steinbach WJ, Cramer Jr RA, Perfect BZ, Asfaw YG, Sauer TC, Najvar LK, Kirkpatrick WR, Patterson TF, Benjamin DK, Jr., Heitman J et al. Calcineurin controls growth, morphology, and pathogenicity in Aspergillus fumigatus. Eukaryot Cell. 2006:5(7):1091-103.

23. Juwvadi PR, Lamoth F, Steinbach WJ. Calcineurin as a multifunctional regulator: unraveling novel functions in fungal stress responses, hyphal growth, drug resistance, and pathogenesis. Fungal Biol Rev. 2014;28(2-3):56-69.

24. Wibawa T, Nurrokhman, Baly I, Daeli PR, Kartasasmita G, Wijayanti N. Cyclosporine A decreases the fluconazole minimum inhibitory concentration of Candida albicans clinical isolates but not biofilm formation and cell growth. Trop Biomed. 2015;32(1):176-82.

25. Sherry L, Rajendran R, Lappin DF, Borghi E, Perdoni F, Falleni M, Tosi D, Smith K, Williams C, Jones B et al. Biofilms formed by Candida albicans bloodstream isolates display phenotypic and transcriptional heterogeneity that are associated with resistance and pathogenicity. BMC Microbiol. 2014;14:182

26. Luo G, Samaranayake LP. Candida glabrata, an emerging fungal pathogen, exhibits superior relative cell surface hydrophobicity and adhesion to denture acrylic surfaces compared with Candida albicans. APMIS. 2002; 110(9):601-10.

27. Pompilio A, Piccolomini R, Picciani C, D'Antonio D, Savini V, Di Bonaventura $G$. Factors associated with adherence to and biofilm formation on 
polystyrene by Stenotrophomonas maltophilia: the role of cell surface hydrophobicity and motility. FEMS Microbiol Lett. 2008;287(1):41-7.

28. Samaranayake YH, Wu PC, Samaranayake LP, So M. Relationship between the cell surface hydrophobicity and adherence of Candida krusei and Candida albicans to epithelial and denture acrylic surfaces. APMIS. 1995; 103(10):707-13.

29. Bader T, Schroppel K, Bentink S, Agabian N, Kohler G, Morschhauser J. Role of calcineurin in stress resistance, morphogenesis, and virulence of a Candida albicans wild-type strain. Infect Immun. 2006;74(7):4366-9.

30. Reedy JL, Filler SG, Heitman J. Elucidating the Candida albicans calcineurin signaling cascade controlling stress response and virulence. Fungal Genet Biol. 2010;47(2):107-16

31. Kojic EM, Darouiche RO. Candida infections of medical devices. Clin Microbiol Rev. 2004;17(2):255-67.

32. Lynch AS, Robertson GT. Bacterial and fungal biofilm infections. Annu Rev Med. 2008;59:415-28.

33. Blankenship JR, Mitchell AP. How to build a biofilm: a fungal perspective. Curr Opin Microbiol. 2006;9(6):588-94.

34. Dominic RM, Shenoy S, Baliga S. Candida biofilms in medical devices: evolving trends. Kathmandu Univ Med J. 2007:5(3):431-6.

35. Liu R, Chen X, Falk SP, Masters KS, Weisblum B, Gellman SH. Nylon-3 polymers active against drug-resistant Candida albicans biofilms. J Am Chem Soc. 2015;137(6):2183-6.

36. Liu Y, Filler SG. Candida albicans Als3, a multifunctional adhesin and invasin. Eukaryot Cell. 2011;10(2):168-73.

37. Nobile CJ, Schneider HA, Nett JE, Sheppard DC, Filler SG, Andes DR, Mitchell AP. Complementary adhesin function in C. albicans biofilm formation. Curr Biol. 2008;18(14):1017-24.

38. Finkel JS, Xu W, Huang D, Hill EM, Desai JV, Woolford CA, Nett JE, Taff H, Norice CT, Andes DR, et al. Portrait of Candida albicans adherence regulators. PLoS Pathog. 2012;8(2), e1002525.

39. Tronchin G, Pihet M, Lopes-Bezerra LM, Bouchara JP. Adherence mechanisms in human pathogenic fungi. Med Mycol. 2008;46(8):749-72.

40. Nobile CJ, Mitchell AP. Genetics and genomics of Candida albicans biofilm formation. Cell Microbiol. 2006;8(9):1382-91.

41. Li DD, Wang Y, Dai BD, Li XX, Zhao LX, Cao YB, Yan L, Jiang YY. ECM17-dependent methionine/cysteine biosynthesis contributes to biofilm formation in Candida albicans. Fungal Genet Biol. 2013;51:50-9.

42. Jiang C, Dong D, Yu B, Cai G, Wang X, Ji Y, Peng Y. Mechanisms of azole resistance in 52 clinical isolates of Candida tropicalis in China. J Antimicrob Chemother. 2013;68(4):778-85.

43. Cowen LE. The evolution of fungal drug resistance: modulating the trajectory from genotype to phenotype. Nat Rev Microbiol. 2008;6(3):187-98.

44. Anderson JB. Evolution of antifungal-drug resistance: mechanisms and pathogen fitness. Nat Rev Microbiol. 2005;3(7):547-56.

45. Garcia-Sanchez S, Aubert S, Iraqui I, Janbon G, Ghigo JM, d'Enfert C. Candida albicans biofilms: a developmental state associated with specific and stable gene expression patterns. Eukaryot Cell. 2004;3(2):536-45

46. Albertson GD, Niimi M, Cannon RD, Jenkinson HF. Multiple efflux mechanisms are involved in Candida albicans fluconazole resistance. Antimicrob Agents Chemother. 1996;40(12):2835-41.

47. Calabrese D, Bille J, Sanglard D. A novel multidrug efflux transporter gene of the major facilitator superfamily from Candida albicans (FLU1) conferring resistance to fluconazole. Microbiology. 2000;146(Pt 11):2743-54.

48. Marchetti O, Moreillon P, Entenza JM, Vouillamoz J, Glauser MP, Bille J, Sanglard D. Fungicidal synergism of fluconazole and cyclosporine in Candida albicans is not dependent on multidrug efflux transporters encoded by the CDR1, CDR2, CaMDR1, and FLU1 genes. Antimicrob Agents Chemother. 2003:47(5):1565-70.

49. Galan-Ladero MA, Blanco-Blanco MT, Hurtado C, Perez-Giraldo C, Blanco MT, Gomez-Garcia AC. Determination of biofilm production by Candida tropicalis isolated from hospitalized patients and its relation to cellular surface hydrophobicity, plastic adherence and filamentation ability. Yeast. 2013;30(9):331-9

50. Kanoshiki RL, de Paula SB, Santos JP, Morey AT, Souza NB, Yamauchi LM, Dias Filho BP, Yamada-Ogatta SF. Effects of fluconazole treatment of mice infected with fluconazole-susceptible and -resistant Candida tropicalis on fungal cell surface hydrophobicity, adhesion and biofilm formation. Indian J Med Microbiol. 2015;33(Suppl):97-101.
51. Kolecka A, Chorvat Jr D, Bujdakova H. The impact of growth conditions on biofilm formation and the cell surface hydrophobicity in fluconazole susceptible and tolerant Candida albicans. Folia Microbiol. 2015;60(1):45-51.

52. Silva-Dias A, Miranda IM, Branco J, Monteiro-Soares M, Pina-Vaz C, Rodrigues AG. Adhesion, biofilm formation, cell surface hydrophobicity, and antifungal planktonic susceptibility: relationship among Candida spp. Front Microbiol. 2015;6:205.

53. Wang $H$, Liang $Y$, Zhang B, Zheng W, Xing L, Li M. Alkaline stress triggers an immediate calcium fluctuation in Candida albicans mediated by Rim101p and Crz1p transcription factors. FEMS Yeast Res. 2011;11(5):430-9.

54. Karababa M, Valentino E, Pardini G, Coste AT, Bille J, Sanglard D. CRZ1, a target of the calcineurin pathway in Candida albicans. Mol Microbiol. 2006;59(5):1429-51.

55. Thomas E, Sircaik S, Roman E, Brunel JM, Johri AK, Pla J, Panwar SL: The activity of RTA2, a downstream effector of the calcineurin pathway, is required during tunicamycin-induced ER stress response in Candida albicans. FEMS Yeast Res. 2015; 15(8). doi: 10.1093/femsyr/fov095.

56. Onyewu C, Wormley Jr FL, Perfect JR, Heitman J. The calcineurin target, Crz1, functions in azole tolerance but is not required for virulence of Candida albicans. Infect Immun. 2004;72(12):7330-3.

57. Cruz LF, Cobine PA, De La Fuente L. Calcium increases Xylella fastidiosa surface attachment, biofilm formation, and twitching motility. Appl Environ Microbiol. 2012;78(5):1321-31.

58. Li DD, Zhao LX, Mylonakis E, Hu GH, Zou Y, Huang TK, Yan L, Wang Y, Jiang YY. In vitro and in vivo activities of pterostilbene against Candida albicans biofilms. Antimicrob Agents Chemother. 2014;58(4):2344-55.

59. Yu LH, Wei X, Ma M, Chen XJ, Xu SB. Possible inhibitory molecular mechanism of farnesol on the development of fluconazole resistance in Candida albicans biofilm. Antimicrob Agents Chemother. 2012;56(2):770-5.

60. Jose A, Coco BJ, Milligan S, Young B, Lappin DF, Bagg J, Murray C, Ramage G. Reducing the incidence of denture stomatitis: are denture cleansers sufficient? J Prosthodont. 2010;19(4):252-7.

61. Pierce CG, Uppuluri P, Tristan AR, Wormley Jr FL, Mowat E, Ramage G, Lopez-Ribot JL. A simple and reproducible 96-well plate-based method for the formation of fungal biofilms and its application to antifungal susceptibility testing. Nat Protoc. 2008;3(9):1494-500.

62. Shinde RB, Chauhan NM, Raut JS, Karuppayil SM. Sensitization of Candida albicans biofilms to various antifungal drugs by cyclosporine A. Ann Clin Microbiol Antimicrob. 2012;11:27.

63. Marchetti O, Moreillon P, Glauser MP, Bille J, Sanglard D. Potent synergism of the combination of fluconazole and cyclosporine in Candida albicans. Antimicrob Agents Chemother. 2000;44(9):2373-81.

64. Ding X, Liu Z, Su J, Yan D. Human serum inhibits adhesion and biofilm formation in Candida albicans. BMC Microbiol. 2014;14:80.

65. Pfaffl MW. A new mathematical model for relative quantification in real-time RT-PCR. Nucleic Acids Res. 2001;29(9), e45.

66. Yoshijima Y, Murakami K, Kayama S, Liu D, Hirota K, Ichikawa T, Miyake Y. Effect of substrate surface hydrophobicity on the adherence of yeast and hyphal Candida. Mycoses. 2010;53(3):221-6.

\section{Submit your next manuscript to BioMed Central and we will help you at every step:}

- We accept pre-submission inquiries

- Our selector tool helps you to find the most relevant journal

- We provide round the clock customer support

- Convenient online submission

- Thorough peer review

- Inclusion in PubMed and all major indexing services

- Maximum visibility for your research

Submit your manuscript at www.biomedcentral.com/submit 